\title{
Zur Emanzipation „der“ muslimischen Frau Kontroversen im Kontext kultureller und patriarchaler Dominanz
}

\author{
Birgit Rommelspacher
}

In der Auseinandersetzung mit „dem“ Islam ist die Frauenfrage zu einem zentralen Prüfstein geworden. Die Gleichberechtigung der Frauen gilt als Voraussetzung für die Integrationsfähigkeit „des“ Islam in die Gesellschaft, denn andernfalls würde der gemeinsame Wertekonsens aufgekündigt und der Zusammenhalt bedroht. Zugleich hat sich jedoch in weiten Kreisen die Meinung festgesetzt, dass „der“ Islam gar nicht modernisierungsfähig sei. Er habe nicht, wie das Christentum durch Reformation und Aufklärung die Menschenrechte in sich aufgenommen. Und den Beweis dafür sehen sie nun vor allem in der Position der Frau, die im Islam generell als unterdrückt gilt.

Diese Einschätzung wird auch nicht irritiert durch die vielen selbstbewussten als Muslima apostrophierten Frauen, die in den Medien, in Politik und Wissenschaft inzwischen auch in Deutschland aktiv sind und zunehmend auch öffentlich sichtbar werden; aber auch nicht durch die zahlreichen Forschungen, die den Muslima in Deutschland eine ebenso starkes Interesse an Gleichberechtigung attestieren wie ihren nicht-muslimischen Zeitgenossinnen. ${ }^{1}$ Als Gegenbeispiele werden dem vielmehr gewalttätige Vorfälle aus meist sehr traditionellen, bildungsfernen Schichten gegenübergestellt, aus Familien, die vielfach wenig Ressourcen haben, um die Herausforderungen der Migration angemessen zu bewältigen - oder auch Beispiele spektakulärer Gewalttaten gegenüber Frauen in sehr unterschiedlichen Ländern und Kulturen. Diese verstellen jedoch den Blick auf die bundesrepublikanische Normalität.

Dabei fragt sich jedoch, warum interessieren sich überhaupt so viele, auch konservative Kreise, für die Emanzipation der Frauen, sobald es sich um Mus-

\footnotetext{
${ }^{1}$ Vgl. Leoni Herwatz-Emden 2003, Yasemin Karakasoglu-Aydin 1998; Gritt M. Klinkhammer, 1999; Frank Jessen und Ulrich Wilamowitz-Moellendorff 2006; Sigrid Nökel 1999.
} 
liminnen handelt? Und: In welchem Verhältnis steht dies Interesse zur Emanzipation der Frauen der Mehrheitsgesellschaft?

\section{Der Blick auf „die“ muslimische Frau}

Inzwischen gibt es eine große Zahl von Analysen, die zeigen, welches Bild in den Pressemedien wie auch in der wissenschaftlichen Literatur oder auf dem Sachbuchmarkt über die „muslimische“ Frau verbreitet wird. ${ }^{2}$ Fast durchgehend wird hier ein Bild gezeichnet, das die muslimischen Frauen als unterdrückt und ohne eigene Subjektivität darstellt. Nur vereinzelt wird auch aus Sicht der muslimischen Frauen selbst berichtet. Das gilt jedoch nicht für die muslimischen Frauen, die das Bild von der unterdrückten Muslima bestätigt haben. So wurde Autorinnen wie Necla Kelek und Seyran Ateş in der deutschen Debatte besonders viel Aufmerksamkeit geschenkt. Ihnen wird auch aufgrund ihrer Herkunft eine besondere Glaubwürdigkeit zugesprochen. Dabei vertritt vor allem Necla Kelek eine stark polarisierende Position: Sie konzentriert sich in ihren Publikationen vornehmlich auf extreme Formen der Gewalt wie Zwangsheirat und Ehrenmorde und zeichnet dabei ein recht grobschlächtiges Bild von „dem“ muslimischen Geschlechterverhältnis, indem gewalttätige Männer ohnmächtigen Frauen gegenübergestellt werden. ${ }^{3}$ In solch polarisierende Bilder passt jedoch z. B. nicht die Tatsache, dass auch Männer Opfer von Zwangsverheiratung werden, ebenso wenig wie die Tatsache, dass vielfach auch Frauen an der Durchsetzung dieser Heiraten beteiligt sind. Frauen können, wie die feministische Debatte der letzten Jahrzehnte zeigte, Mittäterinnen oder auch Täterinnen sein. Die patriarchalen Machtverhältnisse sind nicht einfach als polare Gegensätze zu verstehen, vielmehr sind sie vielschichtiger und widersprüchlicher. Auch Frauen kommt je nach Kontext Macht zu, was jedoch nicht heißt, dass sie damit die übergreifenden männlichen Machtstrukturen außer Kraft setzen. Insofern sind Männer und Frauen Teil eines Systems mit unterschiedlichen Optionen in unterschiedlichen Kontexten. Gendertheorien versuchen diese Tatsache konzeptionell zu fassen. Insofern fragt sich, ob die Debatte um den Islam nicht unter anderem auch dazu dient, bestimmte überholte Positionen innerhalb der feministischen Diskussion

\footnotetext{
${ }^{2}$ Vgl. etwa eine aktuelle Zusammenstellung in Thorsten Gerald Schneiders 2009.

${ }^{3}$ Vgl. Necla Kelek 2006a und 2006b.
} 
wieder heraufzubeschwören. Es ist, als ob in der islamischen Kultur so archaische Verhältnisse herrschten, dass sie einer differenzierten Betrachtung nicht bedürften.

Nimmt man die Ausführungen von Ateş und Kelek ernst, dann sind die muslimischen Frauen nicht nur Opfer patriarchaler Gewalt, sondern auch Opfer einer seit Jahrhunderten unverändert auf ihnen lastenden Kultur. Sie scheinen im Verließ der Vergangenheit eingeschlossen zu sein. So können sie nur von auBen befreit werden. Der Westen und die christliche Kultur wird zur Retterin der von der Geschichte Vergessenen und von ihrer Kultur Verdammten. Dieser Befreiungspathos ist keineswegs neu, sondern hat eine lange europäische Tradition, gerade auch in Bezug auf die muslimischen Frauen. Schon die christlichen Kreuzfahrer sahen es als eine ihrer nobelsten Aufgaben an, die orientalische Prinzessin zu befreien. Ebenso war die „Entschleierung“ der orientalischen Frauen ein zentraler Kern kolonialer Strategie, wie Leila Ahmed in ihrem Werk zum Feminismus und Islam ausführt. ${ }^{4}$

In der aktuellen Debatte wird das Christentum als selbstverständlich mit der Moderne vereinbar angenommen, während dem Islam ein grundsätzlicher Gegensatz unterstellt wird. Eine Position, die bereits Max Weber mit seiner Religionssoziologie prominent in die sozialwissenschaftliche Diskussion eingeführt hat. ${ }^{5}$ Aus feministischer Sicht ist dies insofern brisant, als damit der christliche Antifeminismus stillschweigend akzeptiert wird. Insofern könnte man vermuten, dass die Kritik, die gegenüber dem Christentum unterschlagen wird, sich nun umso heftiger gegen den Islam wendet. Von ihm wird Säkularität eingefordert und an ihm die Behauptung der Unmöglichkeit von Emanzipation und Religion durchexerziert. Eine ähnliche Diskussion gab es im Übrigen bereits in den 80er Jahren des letzten Jahrhunderts, als damals dem Judentum vorgeworfen wurde, mit dem Feminismus unvereinbar zu sein. ${ }^{6}$

Diese Diskussionen machen deutlich, dass es bei der Forderung nach Frauenemanzipation nie nur um patriarchale Machtverhältnisse geht, sondern im-

\footnotetext{
${ }^{4}$ Vgl. Leila Ahmed 1992.

${ }^{5}$ Vgl. Max Weber 1920/1963.

${ }^{6}$ Vgl. etwa die damals sehr populäre Veröffentlichung von Franz Alt 1989; ausführlicher dazu: Birgit Rommelspacher, Antisemitismus und Frauenbewegung in Deutschland, in Brigitte Fuchs und Gabriele Habinger (Hrsg.), Rassismen und Feminismen, Wien 1996.
} 
mer auch die Kultur beziehungsweise Religion mehr oder weniger bewusst mitgedacht wird. Patriarchale und kulturelle Machtverhältnisse scheinen unauflösbar miteinander verwoben zu sein. Das hat auch Auswirkungen auf den hier verwendeten Emanzipationsbegriff, der insofern auch als ein spezifisch christlichsäkularer zu verstehen ist, als er sich als ein Gegenmodell zum muslimischen Geschlechterkonzept versteht.

\section{Welche Emanzipation? Differenz und Gleichheit im Geschlechterverhältnis}

Das muslimische Geschlechterverhältnis scheint dem liberalen westlichen diametral zu widersprechen, betont es doch vor allem die Unterschiedlichkeit der Geschlechter und markiert diese deutlich - wie etwa durch das Kopftuch. Das provoziert. So schreibt etwa Alice Schwarzer: „Wir haben in Deutschland [...] einen Schulterschluss zwischen religiösen Fundamentalisten und westlichen Differenzialistinnen quer durch alle Lager, die beide nichts halten von den universellen Menschenrechten“.7 Mit westlichen „Differenzialistinnen“ meint sie Vertreterinnen von Geschlechtermodellen, die von unterschiedlichen Rollen von Frauen und Männer ausgehen. Solche Modelle sind vor allem liberalen Feministinnen ein Dorn im Auge, da es ihnen gerade darum geht, die Differenzen im Geschlechterverhältnis aufzuheben; denn sobald die Geschlechterrollen als unterschiedliche definiert werden, so die Annahme, setzt sich entlang dieser Unterscheidung die Ungleichbehandlung der Frauen durch. Hier fallen die beiden Bedeutungen von Diskriminierung unmittelbar zusammen: Unterscheidung bedeutet gleichzeitig immer auch Herabsetzung.

Allerdings ist dennoch die Vorstellung von der Unterschiedlichkeit der Geschlechter in unserer Gesellschaft weit verbreitet. In vielen konservativen und insbesondere auch christlichen Kreisen ist die Annahme einer unterschiedlichen Wesenheit von Mann und Frau selbstverständlich. Allerdings wird dies von Feministinnen in der Regel hingenommen und nicht weiter zum Gegenstand politischer Auseinandersetzungen gemacht. Gleichzeitig wird jedoch das muslimische Modell angegriffen. Man könnte vermuten, dass es sich um eine Verschiebung handelt, insbesondere dann, wenn man mit dem muslimischen

\footnotetext{
${ }^{7}$ Alice Schwarzer 2002, S. 134.
} 
Kopftuch nichts anderes als ein konservatives Frauenbild in einem christlichsäkularen Sinn verbindet. Tatsächlich steht es jedoch in einer anderen Tradition und hat, wie wir sehen werden, auch für die heutigen jungen Muslima eine andere Bedeutung.

Die muslimische Konzeption von der Geschlechterdifferenz wird jedoch nicht nur deshalb angegriffen, weil sie als Ersatz für die Bekämpfung konservativer Konzeptionen in der Mehrheitsgesellschaft fungieren kann, sondern auch, weil sich selbst liberale Feministinnen bei der Frage nach Gleichheit und Differenz im Geschlechterverhältnis nicht einig sind, denn einerseits wird Gleichheit angestrebt, andererseits aber ist die Differenz immer auch Quelle von Kritik und Widerstand.

Die patriarchalen Strukturen bedingen unterschiedliche Lebensverhältnisse und damit auch teilweise unterschiedliche Perspektiven von Frauen und Männern. Ohne eine Distanznahme in der Rückbesinnung auf diese Unterschiede würde frau sich ganz dem männlichen Lebensentwurf unterwerfen. So erklärt sich, warum oft gerade Feministinnen die Unterschiede nicht nur betonen, sondern sie mithilfe von Frauenräumen und in Form spezifischer Frauenkulturen immer wieder neu herstellen. In diesem Sinn ist Differenz sowohl Ausdruck von Diskriminierung als auch Ausdruck von Eigenständigkeit. Das bedeutet, dass das Thema Geschlechterunterschiede komplex und in sich widersprüchlich ist. Nun begegnet uns in der islamischen Frau, die das Kopftuch trägt, eine Position, die ohne Umschweife die Verschiedenheit der Geschlechter betont. Diese Betonung von Unterschieden rührt also an einen schwierigen und ungeklärten Punkt in der westlichen Debatte.

Unabhängig davon, ob der Skandalisierung der muslimischen Geschlechterdifferenz eigene Widersprüche zugrunde liegen oder ob damit stellvertretend das christlich konservative Geschlechtermodell bekämpft wird, sicherlich birgt die Orientierung an der Geschlechterdifferenz die Gefahr, Diskriminierungen festzuschreiben. Das Problem ist nur, dass dies ebenso auch bei dem Modell der Geschlechtergleichheit der Fall ist. Denn in der Gleichheit steckt das Moment der Angleichung, was aufgrund der patriarchalen Machtverhältnisse in der Regel die Angleichung der Frauen an das Modell Mann bedeutet. Wenn also etwa Frauen im Beruf genauso „ihren Mann“ stehen wollen, wie dies Männer tun, 
dann tun sie dies in der Regel auf Kosten ihrer Zwei- und Dreifachbelastung, zumindest wenn sie Kinder haben - wie in diesen Tagen die Ergebnisse des sogenannten Bildungspanels erneut bestätigt haben. ${ }^{8}$ Insofern kann nicht einfach das eine Geschlechtermodell als befreiend dem andern als repressiv entgegengesetzt werden. Hilfreicher wäre es, sich mit den Widersprüchlichkeiten beider Modelle auseinander zu setzen, um die jeweiligen Chancen und Gefahren je nach Kontext einschätzen zu können. Eine solche Einschätzung muss die unterschiedlichen Kontexte, in denen Frauen leben, in die Überlegungen mit einbeziehen. Dabei spielt für die Handlungsfähigkeit der Frauen die patriarchale Unterdrückung nicht die einzig Rolle, sondern ebenso wichtig sind ökonomische, politische und kulturelle Machtverhältnisse.

\section{Perspektiven von muslimischen Frauen}

Die Vorstellung, es gäbe „die“ muslimische Stimme in Bezug auf das Geschlechterverhältnis, ist ebenso abwegig wie die, dass es „den“ Islam gäbe. Alleine zum Thema Kopftuch gibt es von Seiten der Muslima sehr viel unterschiedliche Positionen - wie etwa der Sammelband zur „Politik ums Kopftuch“ zeigt. ${ }^{9}$ Das ist auch nicht verwunderlich, denn bei den Muslima gibt es, wie in allen anderen Religionen auch, viele, die kaum religiös sind, solche, die eher konventionell religiös sind, wieder andere, die intensiv gläubig sind oder auch solche, die sich über die Religion politisch radikalisieren und schließlich auch solche, die in verschiedenen Phasen ihres Lebens unterschiedliche Positionen zur Religion einnehmen. Ebenso ist es nicht unerheblich, auf welche politischen und ethnischen Kontexte sie sich dabei beziehen. So gibt es viele aus dem Iran geflüchtete Frauen, die sich vehement gegen das Kopftuch aussprechen, da sie dies mit dem totalitären Regime in ihrer Heimat und mit furchtbaren Erfahrungen von Verfolgung und Unterdrückung verbinden. Andere wiederum sind von den Auseinandersetzungen in der Türkei zwischen Laizismus und Islamismus geprägt.

Neben dem Einfluss von politischen Verhältnissen ist es aber auch eine Frage des religiösen Standorts, der die Position der Frauen bestimmt. Und auch hier

\footnotetext{
${ }^{8}$ Interview mit P. Blossfeld in ZEIT Nr. 33 vom 09.08.2012: Sobald das erste Kind kommt, treten fast automatisch die traditionellen Strukturen in Kraft: In $85 \%$ der Ehen erledigt die Frau die komplette Hausarbeit, auch wenn sie hoch qualifiziert und berufstätig ist.

${ }^{9}$ Vgl. Frigga Haug und Katrin Reimer 2005.
} 
gibt es eine ganze Bandbreite unterschiedlicher Auffassungen auch bei den Frauen, die sich selbst als Feministinnen bezeichnen. So sehen islamische Feministinnen wie etwa Leila Ahmed die Bedeutung des Islam vor allem in seinem ethischen Egalitarismus, der Frauen und Männer dieselbe Würde zuerkennt. ${ }^{10}$ Sie sind zwar verschieden, aber gleichwertig. Dementsprechend gibt es klare Rollenabsprachen, die den Frauen und Männern gleichermaßen, wenn auch unterschiedliche Rechte und Pflichten auferlegen. Das Prinzip der Geschlechtertrennung und der Grundsatz der Verschiedenheit muss ihrer Meinung nach nicht repressiv sein, wenn die Aufgabenteilung ausgeglichen ist. Für solche reformorientierte Feministinnen, für die auch Fatima Mernissi ein prominentes Beispiel ist, ${ }^{11}$ wurde der Koran aufgrund der über Jahrhunderte vorherrschenden patriarchalen Machtverhältnisse einseitig übersetzt und interpretiert. Deshalb gelte es, ihn heute neu zu lesen. Demgegenüber gehen radikale Feministinnen davon aus, dass der Koran selbst den Primat des Mannes festschreibe und es deshalb auch nicht genüge, ihn neu zu interpretieren, sondern dass er in Teilen auch neu formuliert werden muss.

Dem stehen wiederum islamistische Feministinnen gegenüber, für die der Koran wesentlich auf die Gleichstellung der Geschlechter ausgerichtet ist. Sie sehen in den herkömmlichen Auslegungen die Frauenrechte hinreichend berücksichtigt. In ihrem Dogmatismus sind sie den eingangs angeführten antimuslimischen Positionen in Deutschland oft nicht unähnlich. Sie sehen wie diese die Probleme meist bei den anderen: So sind diese Islamistinnen davon überzeugt, dass die Unterdrückung der Frauen im Wesentlichen ein Resultat des Kapitalismus und westlicher Ideologie sei. Die Frauen würden hier ausgebeutet und versklavt, zum Sexobjekt degradiert und der öffentlichen Belästigung preisgegeben. Die Zwänge der Konsumindustrie machten die Frauen zu ihrem wehrlosen Objekt. Zudem habe die „Frauenemanzipation“ ihnen nur Mehrbelastungen eingebracht, die jede Form der Selbstbestimmung unterlaufe. ${ }^{12}$ Das Kopftuch wird von ihnen als ein Medium der Emanzipation im Sinne der Bewahrung der Wür-

\footnotetext{
${ }^{10}$ Vgl. Leila Ahmed 1992.

${ }^{11}$ Vgl. Fatima Mernissi 1989 und 1992.

${ }^{12}$ Vgl. Haideh Moghissi 1999.
} 
de der Frau verstanden. Für sie ist der Islam die Lösung, da ihrer Auffassung nach im Koran die Frau dem Mann gleichgestellt ist.

Über diese verschiedenen Strömungen hinweg konstatiert Göle, dass ein wesentlicher Unterschied zwischen westlich und muslimisch geprägten Emanzipationsvorstellungen der ist, dass die Musliminnen sich sehr viel stärker auf eine Tradition der Geschlechtertrennung beziehen und diese in ihrem Sinn weiterentwickeln. ${ }^{13}$ Die Differenz soll aufrechterhalten und zugleich Gleichheit hergestellt werden. Wie dies zu geschehen habe, auch dazu gehen die Meinungen weit auseinander, vor allem auch in den Auseinandersetzungen zwischen Reformkräften und Traditionalisten innerhalb des Islam, die jedoch vom Westen kaum zur Kenntnis genommen werden.

Wenn wir nun die Situation in Deutschland betrachten, so gibt es inzwischen auch einige Untersuchungen, die die Position der muslimischen Frauen zu erkunden versuchen. Sie konzentrieren sich im Wesentlichen auf Frauen, die ein Kopftuch tragen, weil sie als besonders typisch für eine muslimische Position gelten. Dabei geht es dann, wie etwa in der Untersuchung der Konrad-Adenauer-Stiftung, vor allem darum zu fragen, inwieweit sich diese Frauen in Bezug auf die Gleichstellung von ihren deutschen Altersgenossinnen unterscheiden. Diese Untersuchung zeigt nun, dass es sich in der Mehrzahl um junge, selbstbewusste Frauen handelt, denen etwa die eigene Berufstätigkeit sehr wichtig ist. Ebenso streben sie für sich gleichberechtigte Modelle der Partnerschaft an. „In diesen Ansichten gleichen sie in hohem Maß der deutschen Mehrheitsgesellschaft", resümieren die Autoren. ${ }^{14}$ Diese Musliminnen unterscheiden sich allerdings von ihren anderen muslimischen, christlichen und säkularen Altersgenossinnen darin, dass sie sehr religiös sind und ihren Glauben auch nach außen hin leben wollen. ${ }^{15}$ Die Religion ist ihnen sogar wichtiger als die Familie. Ein relevantes Ergebnis ist jedoch auch, dass sich ein Großteil von ihnen in Deutschland nicht heimisch fühlt:

\footnotetext{
${ }^{13}$ Vgl. Nilüfer Göle 1995.

${ }^{14}$ Frank Jessen und Ulrich von Wilamowitz-Moellendorff 2006, S. 41.

${ }^{15}$ Diese Frauen haben sich für ein Kopftuch entschieden, weil sie damit in erster Linie ihrer religiösen Überzeugung Ausdruck verleihen wollen (90 Prozent). Für nahezu alle der Befragten (98 Prozent) geschah dies aus eigenem Entschluss.
} 
Obwohl die überwiegende Mehrheit von ihnen entweder schon hier geboren wurde oder schon lange hier lebt, ist ihnen Deutschland offenbar fremd geblieben. Sie halten sich für Angehörige einer diskriminierten Minderheit und fühlen sich zurückgesetzt. 80 Prozent der Teilnehmerinnen sind der Ansicht, dass die Türken in Deutschland wie Bürger zweiter Klasse behandelt werden. ${ }^{16}$

Die hohe Religiosität kann also auch als Ausdruck einer eigenständigen kulturellen Verortung verstanden werden.

Das Kopftuch hat also in diesem Kontext nicht nur eine religiöse, sondern zugleich eine kulturelle Bedeutung - die Kopftuchträgerinnnen wollen auch öffentlich und als diskriminierte Minderheit ihren Status in der Gesellschaft behaupten. Das zeigen vor allem auch eine Reihe qualitativer Untersuchungen, die festgestellt haben, dass für viele der befragten Frauen die Religion und damit auch das Kopftuch eine andere Bedeutung als für ihre Vorfahren hat. Ja, sie versuchen sich vielfach gerade durch die Religion von ihren Eltern abzugrenzen und einen individuellen Standort zwischen deren Tradition und der Kultur der Aufnahmegesellschaft zu finden, indem sie einen eigenständigen Bezug zu weltanschaulichen und religiösen Fragen suchen. ${ }^{17}$ Diese so genannten „Neo-Muslima" setzen sich meist sehr gründlich mit dem Islam auseinander und entwickeln differenzierte Strategien der Selbstrepräsentation sowie hohen beruflichen Ehrgeiz und Leistungsbereitschaft. ${ }^{18}$ Mit dem Kopftuch machen die „Töchter der Gastarbeiter"19 ihre Zugehörigkeit zu einer Minderheit öffentlich. Es wird von ihnen also zugleich als ein religiöses und als ein kulturelles Symbol verstanden. Sie machen ihre Differenz freiwillig sichtbar und wandeln so das Stigma in ein Symbol selbstbewusster Identität um.

Das Kopftuch kann damit von einem traditionellen zu einem geradezu modernen Symbol werden, insbesondere wenn man etwa die Frauen betrachtet, die das Kopftuch mit „westlicher“ Kleidung kombinieren und sich teilweise höchst

\footnotetext{
${ }^{16}$ Frank Jessen und Ulrich von Wilamowitz-Moellendorff 2006, S. 19.

${ }^{17}$ Vgl. Yasemin Karakasoglu-Aydin 1998; Sigrid Nökel 1999a und 1999b; Gritt M. Klinkhammer 1999.

${ }^{18}$ Vgl. Sigrid Nökel 1999a, S. 200. Die Reaktivierung kultureller Traditionsbestände im Interesse eines eigenständigen kulturellen Selbstausdrucks finden wir im Übrigen heutzutage vermehrt auch bei anderen Religionen und Kulturen wie etwa bei Juden und Jüdinnen oder bei Menschen mit Vorfahren aus afrikanischen Ländern, aber auch bei Mitgliedern der Mehrheitsgesellschaft, wenn wir etwa an die Reaktivierung von regionalen Dialekten und Traditionen denken.
}

${ }^{19}$ Sigrid Nökel 2002. 
bunt und modebewusst kleiden. Diesen Typus hat Reyhan Sahin in ihrer Untersuchung zur Bedeutung des Kopftuchs für muslimische Kopftuchträgerinnen als „vestimentäres Mixing“ bezeichnet. ${ }^{20}$ Meist pflegen diese Frauen einen ästhetisch sehr anspruchsvollen, sorgfältig ausgewählten Kleidungstil und kombinieren unterschiedliche Kleidungstypen miteinander. Eine dieser Frauen etwa hat ihr Kopftuch im Nacken gebunden und darüber eine Baseballkappe aufgesetzt. Dazu trägt sie eine graue Baggy Jogginghose, die von Anhängern der Hip-Hop-Kultur beabsichtig in Übergrößen getragen werden. Dazu kombiniert sie ein enges T-Shirt mit bunten Motiven. ${ }^{21}$ Eine andere Frau beschreibt ihren Kleidungsstil folgendermaßen:

Also wenn man meine Kleidung im Kleiderschrank sehen würde, würde man sagen, das passt nicht. Ich habe Outfits, die sind ... du würdest sagen richtig bitchlike und dann habe ich Outfits, die sind Hardcore Moslem und dann was ich heute anhabe, so spießig... und dann habe ich auch Outfits, die gehen so mehr in die Gangster Richtung ... also ist alles Mögliche drin ... dann habe ich auch noch so traditionelle Sachen... ${ }^{22}$

Das Interessante ist nun, dass diese Frauen mit ihrem Mixing sowohl bei konventionellen Muslimen als auch bei Angehörigen der Mehrheitsgesellschaft auf Ablehnung stoßen. Beide scheint zu irritieren, dass sie weder in die eine noch in die andere Kategorie einzuordnen sind. Das provoziert. Denn diese Frauen spielen mit den Grenzen. Sie verweisen auf Differenzen und heben sie zugleich wieder auf. Damit wehren sie sich gegen ein Entweder-Oder. Sie weisen darauf hin, dass man auch mehrere Identitätsbezüge gleichzeitig haben kann und dass kulturelle Verortungen ständig im Fluss sind. Entscheidend ist auch, dass sie es sind, die ihre Identität markieren. Sie übernehmen die Deutungsmacht und wehren sich damit gegen Zuschreibungen von anderen. Die Provokation liegt also darin, dass diese Frauen Eindeutigkeiten aufbrechen und die Deutungsmacht für sich zurückgewinnen. Darauf müssen nun die konventionellen Muslime wie auch die Angehörigen der Mehrheitsgesellschaft reagieren - entweder mit Abwehr oder aber mit einem sich wandelnden Bild von dem, was sie unter Musliminnen hier und heute verstehen.

\footnotetext{
${ }^{20}$ Vgl. Reyhan Sahin 2012.

${ }^{21}$ Vgl. ebenda, S. 233.

${ }^{22}$ Ebenda, S. 225.
} 
Allerdings sollte auch dieser Weg von jungen Muslima nicht idealisiert werden, stehen sie immer in Gefahr, sowohl von Seiten der Minderheiten wie auch der Mehrheitsgesellschaft vereinnahmt und in ihrer Selbstbestimmung eingeschränkt zu werden. Indem sie traditionelle Symbole der Geschlechterdifferenz benutzen, können sie auch traditionelle patriarchale Vorstellungsmuster stärken, während die Chance darin liegt, Weiblichkeitsvorstellungen und ihre Symbole durch die Kombination mit neuen Symbolen und Praxen zu verändern.

Wie bereits im obigen Abschnitt über Differenz und Gleichheit im Geschlechterverhältnis angesprochen, scheint gegenüber der Betonung der Geschlechterdifferenz der Weg zur Gleichstellung durch deren Überwindung der einfachere zu sein. Aber auch dieser Weg ist riskant. Diese Erfahrung haben muslimische Frauen etwa im Zusammenhang mit der forcierten Säkularisierung der Türkei in den 20er Jahren des letzten Jahrhunderts gemacht, als ihnen die westliche Kleidung von Kemal Atatürk per Dekret aufgezwungen wurde. Damals wurden im Prozess der Modernisierung der Türkei nicht nur Schrift und Sprache, sondern auch Kleidung und Alltagsverhalten von einem Tag auf den anderen an westliche Standards angepasst. Diese Veränderungen drangen bis in die Körperlichkeit: Wurde Schönheit im Orient jahrhundertelang mit weißer Haut, runden Formen, langsamen Bewegungen und langem Haar verknüpft, so trat nun an dessen Stelle das europäische Schönheitsideal der schlanken, energischen, Korsett tragenden Frau mit kurz geschnittenen Haaren. ${ }^{23}$ Diese aktive, städtische, mit Männern verkehrende, berufstätige Frau mit ihrem aufrechten, dynamischen Körper, an dem täglich gearbeitet wird, wurde zu einem Symbol für die Moderne und für die Zugehörigkeit zur Elite. ${ }^{24}$ Dieser Frauentyp nahm nun in der Gesellschaft eine besondere Stellung ein. Ihr wurde dabei aber, wie Göle resümiert

so sehr ihre Geschlechtlichkeit abgesprochen, dass ihr beinahe eine männliche Identität auf aufoktroyiert wurde. Anders ausgedrückt hat die kemalistische Frau zwar den Gesichtsschleier und den Umhang (Tschador, türkisch çarsaf) abgelegt, dafür aber ihre Geschlechtlichkeit ,verhüllt', in der Öffentlichkeit sich selbst eingepanzert, sich 'unberührbar', ,unerreichbar' gemacht. ${ }^{25}$

\footnotetext{
${ }^{23}$ Vgl. Sigrid Göle 1995, S. 83.

${ }^{24}$ Vgl. ebenda, S. 165.

${ }^{25}$ Ebenda, S. 99.
} 
Damit haben die türkischen Frauen zu jener Zeit bereits das durchexerziert, was liberale deutsche Feministinnen heute von Musliminnen fordern. Aber die „Unterwerfung“ unter westliche Normen brachte auch den Türkinnen damals nicht einfach ihre Befreiung, sondern ließ neue Zwänge hervortreten und den Fortschritt für die Frauen zumindest ambivalent erscheinen - war der Zwang zur Verwestlichung doch selbst ein gewaltträchtiger Akt. Demgegenüber kann es wohl nur darum gehen, die Prozesse zu unterstützen, die den Handlungsspielraum der Frauen in ihrem jeweiligen Kontext erweitern und sich auf ihre Vorstellungen von Emanzipation stützen. Das scheint auch deshalb besonders geboten zu sein, da die Forderungen nach Emanzipation der muslimischen Frauen nach westlich-liberalem Zuschnitt in Deutschland bisher zu recht zweifelhaften Ergebnissen geführt hat - und zwar sowohl für die Musliminnen wie auch für die Frauen der Mehrheitsgesellschaft.

\section{Zur Emanzipation von Frauen in der multikulturellen Gesellschaft}

Die Debatten zur Emanzipation der muslimischen Frau hat auf der politischen Ebene bisher vor allem zu repressiven Maßnahmen geführt: Das Kopftuch wurde für Frauen im öffentlichen Dienst in vielen Bundesländern verboten, das Nachzugsalter für Ehefrauen heraufgesetzt, und es wurden Sprachtests als Vorbedingung zum Familiennachzug eingeführt. Noch problematischer ist vielleicht, dass durch die ständige Beschwörung der Unterdrückung der muslimischen Frau in der Gesellschaft ein Bild entstanden ist, das sie tatsächlich in diese Position drängt und sie darin festhält. So haben gerade junge muslimische Frauen deutlich schlechtere Chancen auf dem Arbeitsmarkt und zwar u. a. mit der Begründung, dass sie traditionell und familienorientiert seien und deshalb keinen beruflichen Ehrgeiz entwickeln würden. Demgegenüber gelten die deutschen Frauen als emanzipiert. Da sie mit Intelligenz und beruflicher Kompetenz identifiziert werden, werden sie den anderen Frauen gegenüber vorgezogen. ${ }^{26}$ Dazu kommt die Signalwirkung des Kopftuchverbots im Öffentlichen Dienst, die in-

\footnotetext{
${ }^{26}$ Vgl. Imam Attia und Helga Marburger 2000; zusammenfassend Castro Varela, Maria do Mar und Dimitria Clayton 2003.
} 
zwischen auch in der privaten Wirtschaft zu erheblichen Benachteiligungen für Frauen mit Kopftuch geführt hat. ${ }^{27}$

Dieser Emanzipationsdiskurs hat nicht nur muslimischen Frauen zumindest auf dem Arbeitsmarkt erheblich geschadet, sondern er hat zugleich die soziale Schere zwischen einheimischen und eingewanderten Frauen verstärkt. Denn Frauen der Mehrheitsgesellschaft sind in den letzten Jahrzehnten nicht zuletzt aufgrund der Unterschichtung durch Migrantinnen aufgestiegen. Das heißt, die unteren Ränge, die bei ihrem beruflichen Aufstieg frei wurden, haben nicht deutsche Männer, sondern vielmehr Migrantinnen und Menschen ohne Papiere eingenommen. Auch im Privatbereich ist die Arbeit von Migranten vielfach die Voraussetzung für die Vereinbarkeit von Beruf und Familie für die mittelständischen einheimischen Frauen geworden. Sie können aus der Privatsphäre in die Öffentlichkeit des Erwerbsbereichs treten, denn an ihre Stelle rücken Migrantinnen nach und entlasten sie bei Hausarbeit und Kinderbetreuung.

Die Folge davon ist nicht nur, dass die eingewanderten Frauen geringere Chancen zum sozialen Aufstieg haben, sondern auch, dass die Situation der alteingesessenen deutschen Frauen idealisiert wird. Sie bemessen ihre Emanzipation nicht mehr so sehr an ihrer Position bezüglich der Männer, sondern vielmehr an ihrem Abstand zu den muslimischen Frauen. Es scheint, wie wenn die Geschlechterhierarchie durch eine ethnische Hierarchie kompensiert würde. Emanzipation wird dann zur Illusion, wenn sie nicht auf der Aufhebung der Arbeitsteilung im Geschlechterverhältnis basiert, sondern auf andere Machtverhältnisse ausweicht. Durch den ethnisch bedingten Aufstieg wird die Illusion genährt, dass bezüglich der Emanzipation der Frauen der Mehrheitsgesellschaft kaum mehr Handlungsbedarf bestehe, da die "deutsche Frau“ ja emanzipiert sei. So wird das Geschlechterverhältnis vom Veränderungsdruck entlastet und der Konfliktstoff gewissermaßen ausgelagert. Entsprechend engagiert sind auch plötzlich viele Männer der Mehrheitsgesellschaft in Sachen Gleichberechtigung, geht es doch nun vor allem um die Bestätigung der eigenen „fortschrittlichen“ Verhältnisse.

${ }^{27}$ Vgl. Senatsverwaltung für Integration, Arbeit und Soziales 2008. 


\section{Schluss}

Wollte der Feminismus nicht kulturelle Hierarchien noch verstärken, sondern sich für die Emanzipation aller Frauen einsetzen, müsste er alle Formen von Herrschaft in Frage stellen und mit der Ungleichheit der Geschlechter auch die Hierarchie zwischen den sozialen Klassen, den Ethnien und Religionen hinterfragen. Das war jedoch in der europäischen Frauenbewegung selten der Fall, denn der universalen Solidarität aller Frauen stand immer auch die Loyalität gegenüber der eigenen Klasse, Nation, „Rasse“ oder Religion gegenüber. Insofern gilt es, den Begriff des Feminismus zu kontextualisieren und ihn jeweils kritisch auf seine jeweilige Positionierung zu hinterfragen. Es wäre zu fragen, wer welche Forderungen für wen stellt beziehungsweise wer jeweils daran beteiligt ist. Denn diese Forderungen sind immer an die Position der Sprechenden gebunden. In ihren Visionen vom guten Leben spiegeln sich die eigenen Lebensverhältnisse.

Ohne sich auf die anderen Frauen einzulassen und sich mit ihrer Geschichte der Modernisierung, ihren Kämpfen um Anerkennung und Macht zu befassen, und ohne sich ihre widersprüchlichen Loyalitäten vor Augen zu führen - ohne ein solches sich Einlassen auf die Andere kann wohl kaum davon gesprochen werden, dass man/frau an ihrer Emanzipation interessiert wäre. Und wenn wir den muslimischen Frauen in Deutschland heute zuhören, so ist auf alle Fälle klar, dass ihr Kampf um Anerkennung immer auch die Frage ihrer kulturellen und religiösen Diskriminierung mit umfasst. Sie können die Frage der Frauenbefreiung nicht abgelöst von ihrem kulturellen Kontext sehen.

Auffallend ist jedenfalls, dass trotz des enormen Interesses an der Emanzipation der muslimischen Frauen sich kaum jemand für die Frauenbewegungen in muslimischen Ländern interessiert. Zumindest in Deutschland wird die Literatur zu muslimischen Emanzipationsbewegungen nicht zur Kenntnis genommen. Wichtige Bücher, wie etwa das grundlegende Werk von Leila Ahmed ${ }^{28}$ oder die verschiedene Arbeiten von Margot Badran ${ }^{29}$, werden gar nicht übersetzt. ${ }^{30}$

\footnotetext{
${ }^{28}$ Vgl. Leila Ahmed 1992.

${ }^{29}$ Vgl. zuf. etwa Margot Badran, Feminism in Islam 2010.

${ }^{30}$ Vgl. etwa auch Nima Naghibi 2007.
} 
Demgegenüber wird durch die ständige Beschwörung der Unterdrückung der muslimischen Frauen diese diskursiv reproduziert. Diese Diskussionen reden das herbei, was sie zu bekämpfen vorgeben. In einem aufklärerischen Gestus wird auf die Unmöglichkeit der Emanzipation der Muslima hingewiesen und ihnen damit eine eigene Subjektposition abgesprochen und die vielfältigen Auseinandersetzungen muslimischer Frauen mit der Moderne in den verschiedenen sozialen und kulturellen Kontexten nicht wahrgenommen. Demgegenüber müsste es darum gehen, die muslimischen Frauen selbst zu Wort kommen zu lassen und ihre unterschiedlichen Positionen wahrzunehmen und sich in den verschiedenen Bereichen für ihre tatsächliche Gleichstellung einzusetzen.

\section{Literatur}

Attia, Iman und Marburger, Helga (Hrsg.), Alltag und Lebenswelten von Migrantenjugendlichen, Frankfurt am Main 2000.

Badran, Margot, Feminism in Islam. Oxford 2010.

Ahmed, Leila, Women and Gender in Islam. Historical Roots of a Modern Debate. New Haven \& London 1992.

Alt, Franz, Jesus, der neue Mann. München 1989.

Castro Varela, Maria do Mar und Clayton, Dimitria (Hrsg.), Migration, Gender, Arbeitsmarkt. Neue Beiträge zu Frauen und Globalisierung, Königstein/Taunus 2003.

Göle, Nilüfer, Republik und Schleier, Berlin 1995.

Haug, Frigga und Reimer, Katrin (Hrsg.), Politik ums Kopftuch, Hamburg 2005.

Herwatz-Emden, Leoni (Hrsg.), Einwanderer Familien: Geschlechterverhältnisse, Erziehung und Akkulturation. Göttingen 2003.

Jessen, Frank und Wilamowitz-Moellendorff, Ulrich von Nökel, Das Kopftuch - Die Entschleierung eines Symbols? Sankt Augustin/Berlin 2006.

Karakasoglu-Aydin, Yasemin, „Kopftuch-Studentinnen” türkischer Herkunft an deutschen Universitäten. Impliziter Islamismusvorwurf und Diskriminierungserfahrungen, in: Heiner Bielefeldt und Wilhelm Heitmeyer (Hrsg.): Politisierte Religion Ursachen und Erscheinungsformen des modernen Fundamentalismus (S. 450-473). Frankfurt/Main 1998.

Klinkhammer, Gritt M., Individualisierung und Säkularisierung islamischer Religiosität: zwei Türkinnen in Deutschland, in: Jonker, Gerdien (Hrsg.), Kern und Rand. Religiöse Minderheiten aus der Türkei in Deutschland (S. 221-236). Berlin 1999.

Kelek, Necla, Die fremde Braut. Ein Bericht aus dem Inneren des türkischen Lebens in Deutschland. Köln 2006a. 
Kelek, Necla, Die verlorenen Söhne. Plädoyer für die Befreiung des türkisch-muslimischen Mannes. Köln 2006b.

Mernissi, Fatema, Der politische Harem, Frankfurt am Main 1989.

Mernissi, Fatema, Die Angst vor der Moderne, Hamburg 1992.

Moghissi, Haideh, Feminism and Islamic Fundamentalism, London, New York 1999.

Naghibi, Nima, Rethinking Global Sisterhood: Western Feminism and Iran. Minnesota 2007.

Nökel, Sigrid, Das Projekt der Neuen islamischen Weiblichkeit als Alternative zu Essentialisierung und Assimilierung, in: Jonker, Gerdien (Hrsg.), Kern und Rand. Religiöse Minderheiten aus der Türkei in Deutschland, Berlin 1999a, S. 187-205.

Nökel, Sigrid, Islam und Selbstbehauptung - Alltagsweltliche Strategien junger Frauen in Deutschland, in: Klein-Hesseling, Ruth; Nökel, Sigrid und Werner, Karin (Hrsg.), Der neue Islam der Frauen: Weibliche Lebenspraxis in der globalisierten Moderne. Fallstudien aus Afrika, Asien und Europa, Bielefeld 1999b, S. 124-146.

Nökel, Sigrid, Die Töchter der Gastarbeiter und der Islam. Zur Soziologie alltagsweltlicher Anerkennungspolitiken. Eine Fallstudie, Bielefeld 2002.

Rommelspacher, Birgit, Geschlecht und Migration in einer globalisierten Welt. Zum Bedeutungswandel des Emanzipationsbegriffs. In: Gemende, Marion; Munsch, Chantal und Weber-Unger-Rotino, Steffi (Hrsg.), Eva ist emanzipiert, Mehmet ist ein Macho. Zuschreibung, Ausgrenzung, Lebensbewältigung und Handlungsansätze im Kontext von Migration und Geschlecht. Weinheim/München 2007, S. 49-61.

Rommelspacher, Birgit, Dominante Diskurse. Zur Popularität von „Kultur” in der aktuellen Islam-Debatte, in: Iman, Attia (Hrsg.), Orient- und Islambilder. Interdisziplinäre Beiträge zu Orientalismus und antimuslimischem Rassismus, Münster 2007.

Şahin, R., Die Bedeutung des muslimischen Kopftuchs. Eine kleidungssemiotische Untersuchung muslimischer Kopftuchträgerinnen in der Bundesrepublik Deutschland. Diss. Universität Bremen 2012.

Schneiders Thorsten Gerald (Hrsg.), Islamfeindlichkeit - Wenn die Grenzen der Kritik verschwimmen, Wiesbaden 2009.

Schwarzer Alice (Hrsg.), Die Gotteskrieger und die falsche Toleranz, Köln 2002.

Senatsverwaltung für Integration, Arbeit und Soziales Berlin (Hrsg.), Mit Kopftuch außen vor? Schriften der Landesstelle für Gleichbehandlung - gegen Diskriminierung 2. Berlin 2008.

Tietze, Nikola, Islamische Identitäten Formen muslimischer Religiosität junger Männer in Deutschland und Frankreich. Hamburg 2001.

Weber, Max, Gesammelte Aufsätze zur Religionssoziologie I. Tübingen 1920/1963 (Paul Siebeck). 